2007-09-01

\title{
Resolution Investigation of a Ratiometric Wavelength Measurement System
}

\author{
Qian Wang \\ Technological University Dublin \\ Ginu Rajan \\ Technological University Dublin, ginu.rajan@tudublin.ie \\ Pengfei Wang \\ Technological University Dublin, pengfei.wang@tudublin.ie
}

See next page for additional authors

Follow this and additional works at: https://arrow.tudublin.ie/engscheceart

Part of the Electrical and Computer Engineering Commons

\section{Recommended Citation}

Wang, G., Rajan, G., Wang, P., Farrell, G.:Resolution Investigation of a Ratiometric Wavelength Measurement System. Applied Optics, Vol 46, (25), 2007, pp.6362-6367. doi:10.1364/A0.46.006362

This Article is brought to you for free and open access by the School of Electrical and Electronic Engineering at ARROW@TU Dublin. It has been accepted for inclusion in Articles by an authorized administrator of ARROW@TU Dublin. For more information, please contact arrow.admin@tudublin.ie, aisling.coyne@tudublin.ie, gerard.connolly@tudublin.ie.

Funder: IRCSET

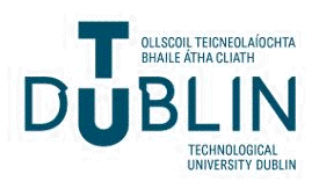


Authors

Qian Wang, Ginu Rajan, Pengfei Wang, and Gerald Farrell

This article is available at ARROW@TU Dublin: https://arrow.tudublin.ie/engscheceart/83 


\title{
Resolution investigation of a ratiometric wavelength measurement system
}

\author{
Qian Wang, * Ginu Rajan, Pengfei Wang, and Gerald Farrell \\ Applied Optoelectronics Centre, School of Electronics and Communications Engineering, Dublin Institute of Technology, \\ Kevin Street, Dublin D8, Ireland \\ *Corresponding author: qian.wang@osa.org
}

Received 16 October 2006; revised 23 May 2007; accepted 2 July 2007; posted 5 July 2007 (Doc. ID 76156); published 27 August 2007

\begin{abstract}
Theoretical analysis and experimental investigations are presented on the resolution of a ratiometric wavelength measurement system. Theoretical modeling indicates that the resolution of a ratiometric wavelength measurement system is determined by the signal-to-noise ratio of the input signal and the noise of the photodetectors associated with optical-to-electronic conversion. For experimental verification, a ratiometric system employing a macrobending standard single-mode fiber is developed and corresponding results are in good agreement with the theoretical prediction. (C) 2007 Optical Society of America OCIS codes: $120.4820,120.4570,060.2380$.
\end{abstract}

\section{Introduction}

Wavelength measurement is required in multichannel dense wavelength division multiplexing (DWDM) optical communication systems and fiberBragg-grating- (FBG-) based optical sensing systems. DWDM requires the accurate setting and maintenance of the transmitter's wavelength [1,2]. A basic FBG-based optical sensing system requires a broadband optical source, the FBG component, and a wavelength demodulation system detecting the wavelength shift of the reflected light, which is crucial in the successful implementation of the above optical sensing techniques [3].

There are various wavelength measurement schemes and among them is the ratiometric detection scheme [4-12]. This technique employs an edge filter and utilizes the transition region of its transmission response, converting the wavelength measurement into the measurement of the ratio of signal intensity. It has a simple configuration and offers a high-speed measurement as compared with, for example, the wavelength-scanning-based active measurement schemes. Recent investigations on ratiometric wavelength measurement are mainly focused on proposing

0003-6935/07/256362-06\$15.00/0

(C) 2007 Optical Society of America different types of the edge filter, such as bulk thinfilm filters [4], bioconical fiber filters [7], bending fiber filters [10], hetero-core fiber filters [11], and so on.

In the previous investigation in [12], the ratiometric system was analyzed in the context of the limited signal-to-noise ratio (SNR) of the input signal, and it was shown that for a given measurable wavelength range, the slope of the edge filter is limited due to the noise of the input signals. In that research, the noise of the photodetectors associated with optical-to-electronic conversion was neglected. However, the noise of photodetectors plays an important role in determining the resolution of the ratiometric wavelength measurement system, i.e., the minimal wavelength shift that the system can detect, which is an important specification in the evaluation of measurement's performance. Therefore, the investigation on the resolution of a ratiometric wavelength measurement system is presented in this paper. In Section 2, theoretical modeling of the ratiometric system and an analysis of the resolution is presented, which shows that, due to the noise of the input signal and photodetectors, the ratiometric system's resolution is limited and the achievable resolution is predicted. In Section 3, a ratiometric system based on a macrobending fiber is developed for the experimental verification. As proposed in [10], the macrobending fiber acts as the edge filter in the wavelength range 
from 1500 to $1600 \mathrm{~nm}$. The measured ratio of the system is investigated for different wavelength shifts of the input signal and different noise of the photodetectors. The demonstration example shows the influence of the noise of the input signal and photodetectors on the system's performance and is in good agreement with the theoretical prediction.

\section{Resolution Analysis of a Ratiometric Wavelength Measurement System}

Figure 1 shows the schematic configuration of a ratiometric wavelength measurement system employing an edge filter. The input signal is split into two signals. One passes through a reference arm and the other passes through the bending fiber. Two photodetectors are placed at the ends of both arms. By measuring the ratio of the electrical outputs of the two photodetectors, the wavelength of the input signal can be determined assuming a suitable calibration has taken place.

For this ratiometric configuration, the output ratio at wavelength $\lambda_{0}$ is

$$
\begin{aligned}
R\left(\lambda_{0}\right) & =-10 \log _{10}\left(\frac{\int P_{1}(\lambda) I_{\lambda_{0}}(\lambda) T_{f}(\lambda) d \lambda}{\int P_{2}(\lambda) I_{\lambda_{0}}(\lambda) d \lambda}\right) \\
& =P_{f}-P_{r},
\end{aligned}
$$

where $P_{1}(\lambda)$ and $P_{2}(\lambda)$ are the outputs of the splitter, and for an ideal case it is assumed that $P_{1}(\lambda)$ $=P_{2}(\lambda)=0.5$ independent of the wavelength in the measurable range. $T_{f}(\lambda)$ is the transmission response of the edge filter, and $I_{\lambda_{0}}(\lambda)$ is the spectrum of the input signal. $P_{f}$ is the output from the arm with an edge filter $\left(P_{f}=-10 \log _{10}\left[\int P_{1}(\lambda) I_{\lambda_{0}}(\lambda) T_{f}(\lambda) d \lambda\right]\right)$ and $P_{r}$ is the output from the reference arm $\left(P_{r}=-10\right.$ $\left.\log _{10}\left[\int P_{2}(\lambda) I_{\lambda_{0}}(\lambda) d \lambda\right]\right)$. The spectrum of the input signal with a peak wavelength $\lambda_{0}$ can be approximated by a Gaussian function with a spectral width $\Delta \lambda$, while considering the limited signal-to-noise ratio simultaneously, as presented in [12], which investigated the influence of the limited signal-to-noise ratio on the design of the edge filter employed in the ratiometric system. Equation 1 does not take account of the noise of the photodetectors. However, the noise of photodetectors associated with optical-to-electronic conversion, plays a crucial role in the resolution of the

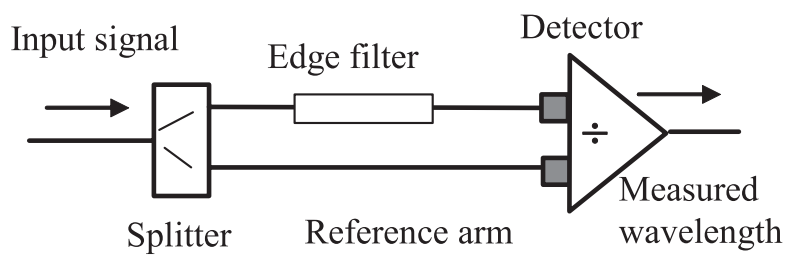

Fig. 1. Configuration of the ratiometric wavelength measurement system employing an edge filter. ratiometric system. To take account of the noise generated by the photodetectors and the electronic circuitry, the output ratio is modeled with a random

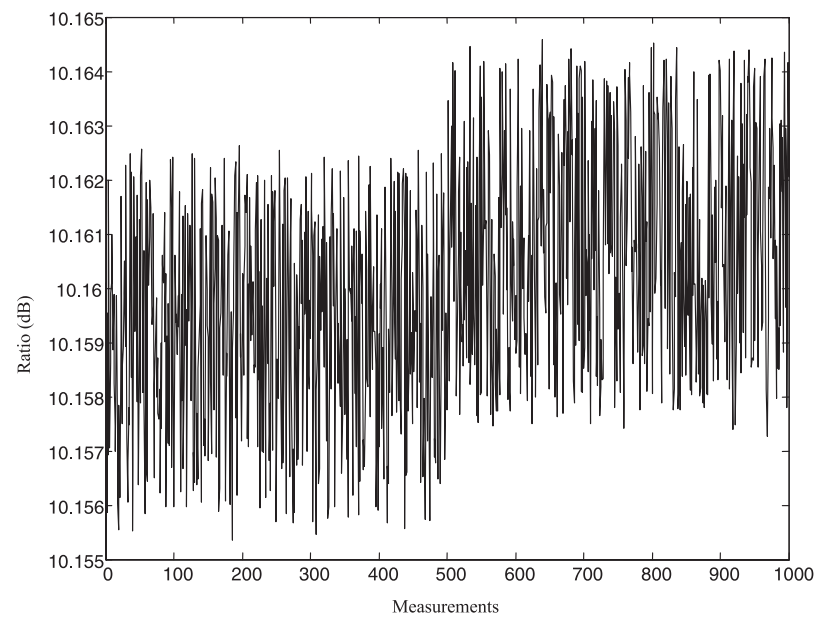

(a)

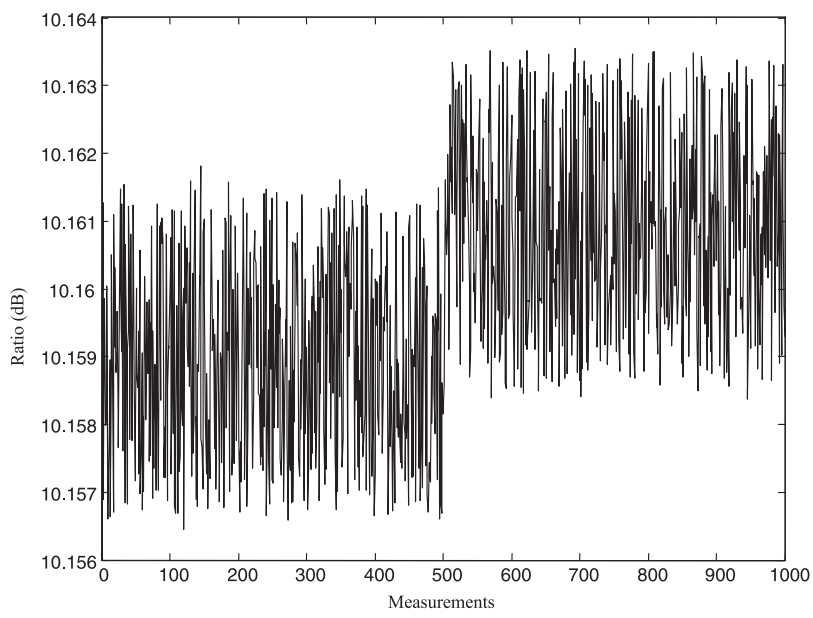

(b)

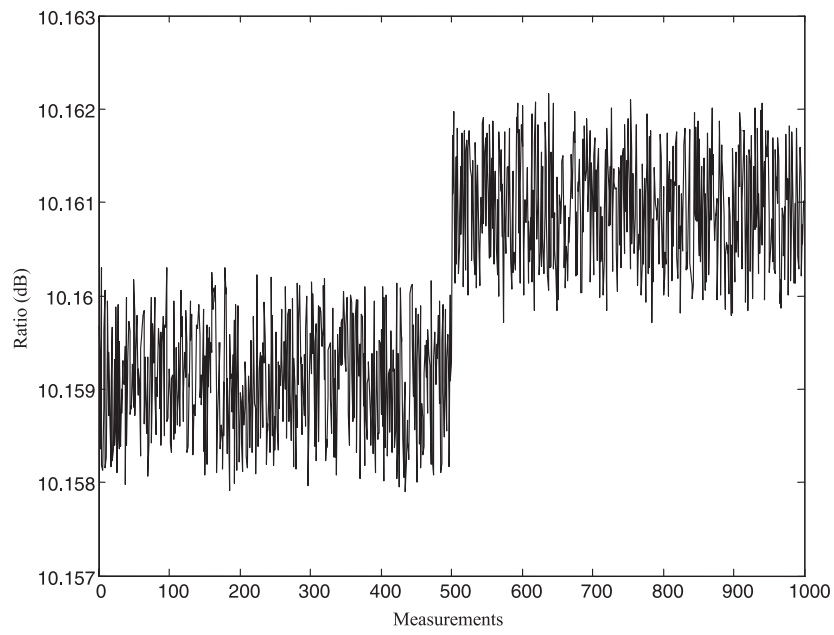

(c)

Fig. 2. Modeled ratios of the system as the tunable laser has a wavelength shift of $10 \mathrm{pm}$ at $1500 \mathrm{~nm}$ for different fluctuation values of the power monitor: (a) $0.007 \mathrm{~dB}$, (b) $0.005 \mathrm{~dB}$, (c) $0.002 \mathrm{~dB}$. 
value number within the resolution range as the following:

$$
R_{r}\left(\lambda_{0}\right)=R\left(\lambda_{0}\right)+r R_{M},
$$

where $R_{M}$ is the range of fluctuation of the output ratio, and $r$ is a random value produced for each measurement within the range $[-0.5,0.5]$.

To illustrate the ratiometric system modeling while considering the noise of the photodetectors, a numerical example is presented in which the output ratio is calculated as the input signal undergoes a wavelength shift. Assume that the input signal from the tunable laser has a wavelength shift of $10 \mathrm{pm}$ at $1550 \mathrm{~nm}$ (the SNR is $50 \mathrm{~dB}$ ) and the corresponding fluctuations $R_{M}$ due to the photodetectors' noise are $0.007,0.005$, and $0.002 \mathrm{~dB}$, respectively (these values are chosen according to the experiment as shown in Section 3). To simplify the problem, the transmission response of the edge filter $\bar{T}_{f}(f)\left(\bar{T}_{f}(f)\right.$ $=-10 \log _{10}\left[T_{f}(\lambda)\right]$ with $\mathrm{dB}$ units) in wavelength range $\left(\lambda_{1}, \lambda_{2}\right)$ is assumed to be a linear function, i.e.,

$$
\bar{T}_{f}(\lambda)=\bar{T}_{f}\left(\lambda_{1}\right)+\frac{\left[\bar{T}_{f}\left(\lambda_{2}\right)-\bar{T}_{f}\left(\lambda_{1}\right)\right]}{\left(\lambda_{2}-\lambda_{1}\right)}\left(\lambda-\lambda_{1}\right) .
$$

For this example, $\lambda_{1}=1500 \mathrm{~nm}, \lambda_{2}=1600 \mathrm{~nm}$, $\bar{T}\left(\lambda_{1}\right)=0$, and $\bar{T}\left(\lambda_{2}\right)=20 \mathrm{~dB}$. The corresponding calculation results are presented in Figs. 2(a)-2(c).

For the measurement system to quantify that there is a wavelength shift requires that the change of the output ratio should be no less than the ratio fluctuation caused by the photodetectors' noise. With the calculated results, it is found that for the above example when the fluctuation of the ratio is less than $0.002 \mathrm{~dB}$, the wavelength shift of $10 \mathrm{pm}$ can be detected clearly through the system output ratio. It seems that, to improve the resolution when the noise of the photodetectors is given, increasing the slope of

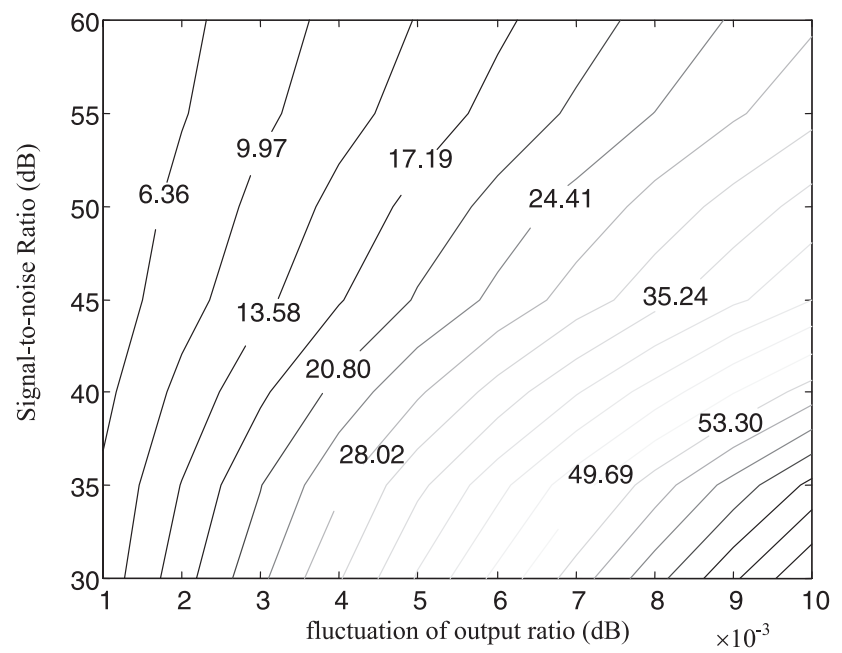

Fig. 3. Contour plot of the theoretical achievable resolution for different SNRs of the input light and fluctuation of the system ratio. the edge filter can be an effective method. However, as investigated in [12], the noise of the input signal puts a limitation on the slope of the edge filter. Therefore, the resolution of the ratiometric system can be determined by the SNR of the input signal and noise of the photodetectors. Take the desired measurable wavelength range from 1500 to $1600 \mathrm{~nm}$ as an example. For different noise levels of the input signal and photodetectors, the achievable resolutions are calculated with the above model. Corresponding results are presented in Fig. 3 (unit of resolution: pm). It can be seen that, when the SNR or fluctuation of the output ratio due to the noise of the photodetectors decreases, the ratiometric system's resolution is improved, and for certain SNRs of the source and fluctuation of the output ratio, picometer resolution can be achieved. For the above numerical example, Fig. 3 shows that when the SNR is $50 \mathrm{~dB}$ and the fluctuations of the ratio are approximately $0.002,0.005$, and $0.007 \mathrm{~dB}$, the corresponding achievable resolutions

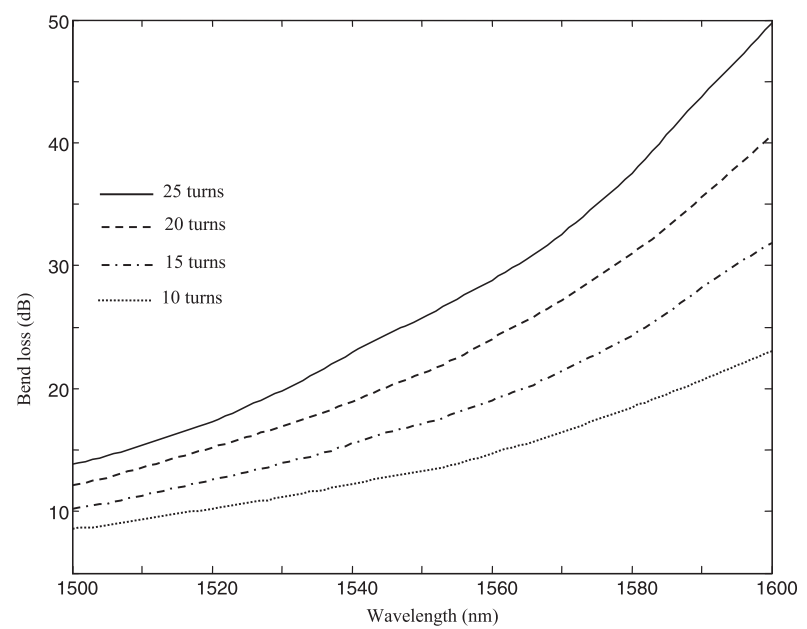

(a)

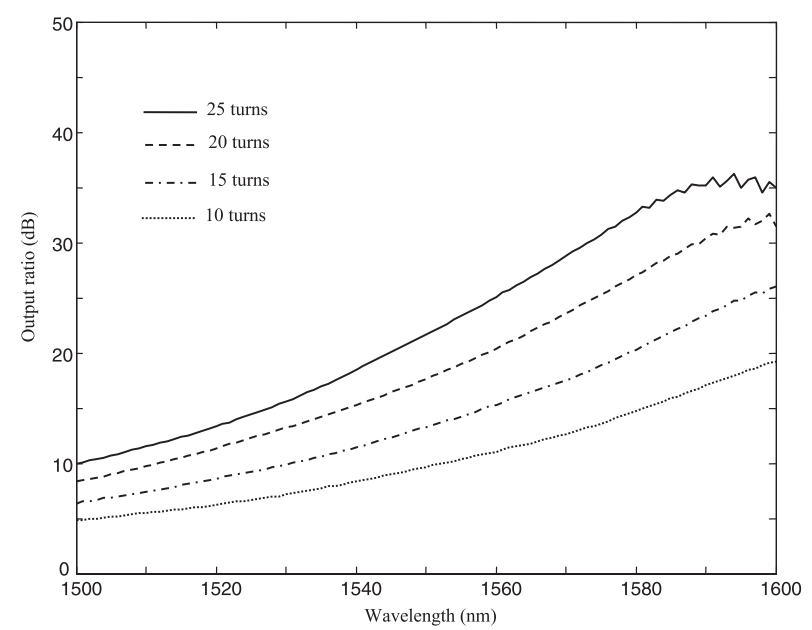

(b)

Fig. 4. (a) Bend loss of a single-mode fiber within the wavelength range from 1500 to $1600 \mathrm{~nm}$ for different bending lengths. (b) Calibration curves for different turns within the wavelength range from 1500 to $1600 \mathrm{~nm}$. 
are approximately 7,18 , and $26 \mathrm{pm}$. To verify this prediction, experimental results are presented in Section 3.

\section{Experimental Results of a Ratiometric System Employing a Macrobending Fiber}

For a macrobending single-mode fiber, most of the previously published investigations are focused on how to predict and lower the bend loss, which is regarded as an adverse effect for light transmission. As proposed in [10], it can also be developed as the edge filter for ratiometric wavelength measurement. Although the length of the bending and bending radius are important parameters in implementing the filtering function, they are not necessary in practice to get the length and radius precisely right, provided a calibration is taken after the system is set up. In our present experiment, a tunable laser and an optical spectrum are used for the measurement of the transmission of the macrobending fiber. As an example, for a bending radius of $10.5 \mathrm{~mm}$, the measured bend losses are presented in Fig. 4(a) with lengths of 10, 15,20 , and 25 turns. The optical filter is not linear over the entire wavelength range from 1500 to 1600 $\mathrm{nm}$, and the spectrum curve becomes steeper for wavelengths over $1550 \mathrm{~nm}$.

For the calibration of the system as shown in Fig. 1 , the above tunable laser and a dual-channel optical powermeter are used. Specifications of the tunable laser will affect the calibration directly, which has also been demonstrated in [12]. In our present calibration experiment, the SNR of the tunable laser is approximately $50 \mathrm{~dB}$. The power stability is $\pm 0.01 \mathrm{~dB}$ for $0 \mathrm{dBm}$ output power. The wavelength stability of

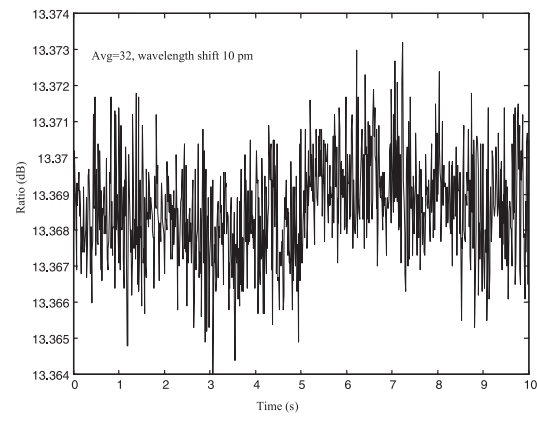

(a)

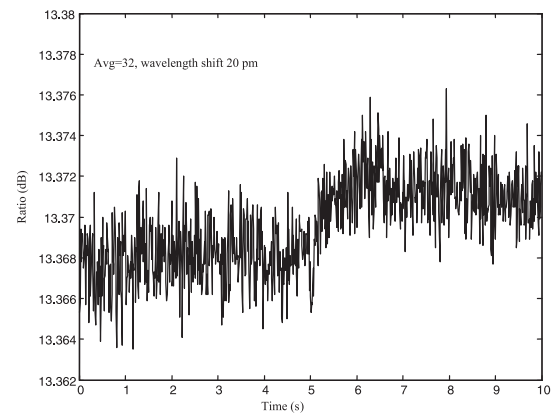

(b)

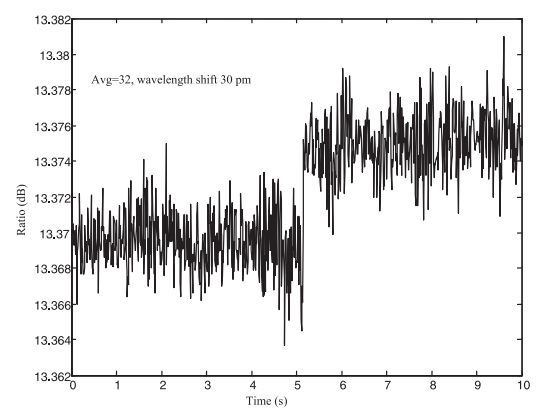

(c)

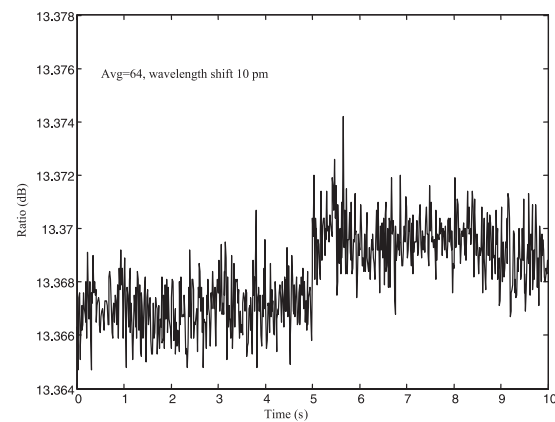

(d)

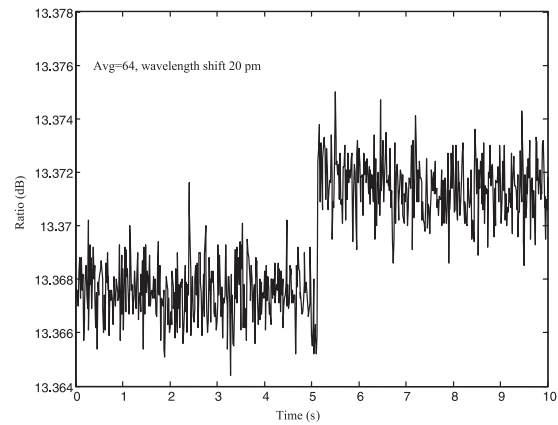

(e)

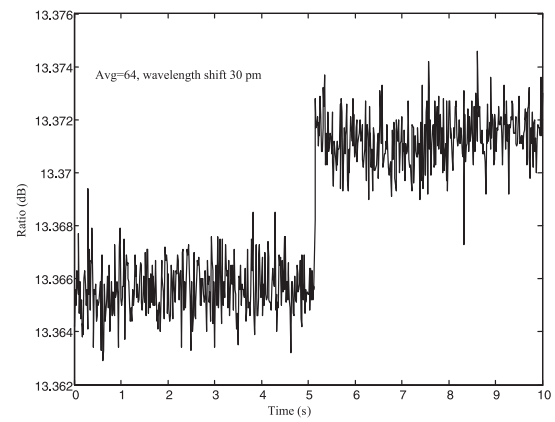

(f)

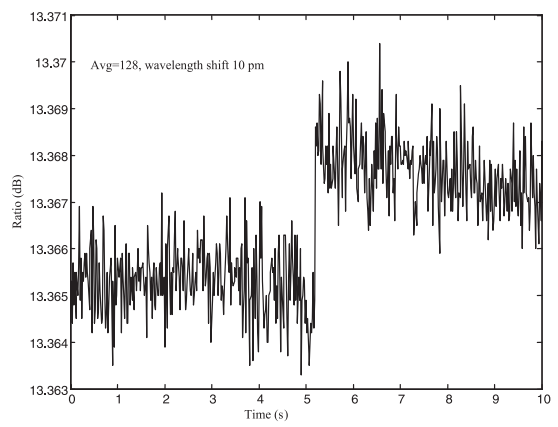

(g)

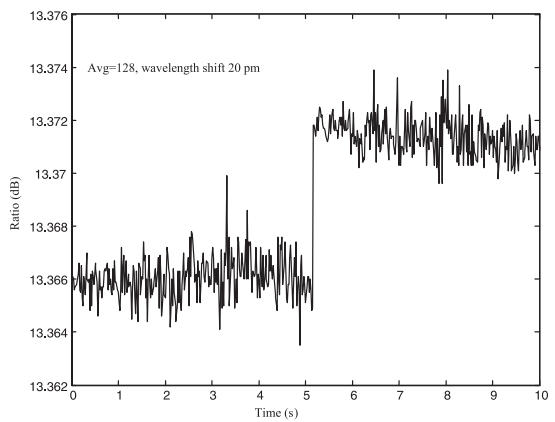

(h)

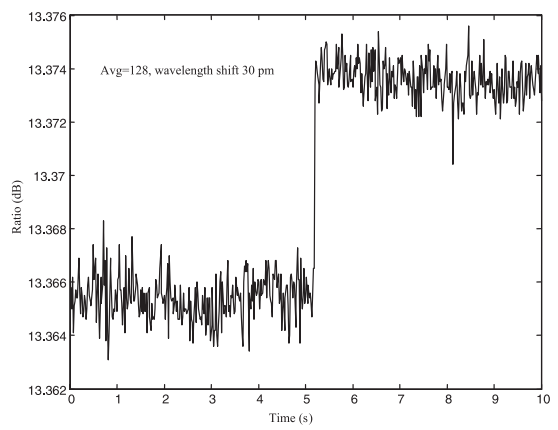

(i)

Fig. 5. Recorded ratios of the system as the tunable laser has a wavelength shift at $1530 \mathrm{~nm}$ for different averaging values of the powermeter: (a) Avg = 32, wavelength shift of $10 \mathrm{pm}$; (b) Avg = 32, wavelength shift of $20 \mathrm{pm}$; (c) Avg = 32, wavelength shift of 30 pm; (d) Avg = 64, wavelength shift of 10 pm; (e) Avg = 64, wavelength shift of 20 pm; (f) Avg = 64, wavelength shift of 30 pm; (g) Avg = 128, wavelength shift of $10 \mathrm{pm}$; (h) Avg = 128, wavelength shift of 20 pm; (i) Avg = 128, wavelength shift of $30 \mathrm{pm}$. 
the tunable laser is $\pm 10 \mathrm{pm}$ at a constant temperature and after $1 \mathrm{~h}$ warming up. The minimum tunable wavelength is $10 \mathrm{pm}$. The dual-channel optical powermeter is a module that can be integrated into a PXI platform and controlled with the developed LabVIEW-based program. It employs two InGaAs photodetectors with a wavelength range from 900 to $1700 \mathrm{~nm}$. The measurable minimum power is -70 $\mathrm{dBm}$ and the maximum power is $10 \mathrm{dBm}$. The fluctuation of the measured ratio can be controlled through setting the averaging value of the dualchannel powermeter. The above bending fiber with the bending radius of $10.5 \mathrm{~mm}$ and bending lengths of $10,15,20$, and 25 turns is used for the calibration. The output ratio of the system is shown in Fig. 4(b). For the bending lengths of 20 and 25 turns, the calibrated plots of the output ratio do not increase monotonically near the upper end of the wavelength range, which is caused by the limited SNR of the input signal.

To show the influence of the noise of the photodetectors on the system resolution experimentally, a wavelength shift from the tunable laser is used and the corresponding measured ratio is recorded to see whether the wavelength shift can be detected. In our experiment, the wavelength shifts used are 10, 20, and $30 \mathrm{pm}$, occupying $5 \mathrm{~s}$ with the measurement period of $10 \mathrm{~s}$ with a starting wavelength of $1530 \mathrm{~nm}$. The bending fiber with 20 turns is chosen, and the slope of the edge filter is approximately $0.202 \mathrm{~dB} / \mathrm{nm}$ at this wavelength. The influence of temperature within this time period is neglected, but it has obvious influence on the system's accuracy for a long-term measurement. The recorded ratios of the system are presented in Figs. 5(a)-5(i) with the averaging values of 32,64 , and 128 of the optical powermeter.

From Figs. 5(a) to 5(c), it can be seen that, when the averaging value is 32 , the wavelength shift cannot be detected due to the random noise (the peakto-peak random fluctuation is approximately 0.007 $\mathrm{dB}$ when the averaging value is 32 ) when the shift values are 10 and $20 \mathrm{pm}$, but it can be detected when the wavelength shift is $30 \mathrm{pm}$. Therefore, the resolution of the ratiometric system is approximately $30 \mathrm{pm}$ when the fluctuation of the ratio is $0.007 \mathrm{~dB}$ and the SNR of the source is approximately $50 \mathrm{~dB}$ (the corresponding theoretical prediction is $26 \mathrm{pm}$ ).

When the averaging value is increased to be 64 , the fluctuation of the ratio is reduced to be approximately $0.005 \mathrm{~dB}$. The recorded ratio is presented from Fig. 5 (d) to5(f) for the wavelength shifts of 10,20 , and 30 $\mathrm{pm}$. When the wavelength shift is $10 \mathrm{pm}$., there is a step change that can be seen, but it is not sufficiently clear; however, when the wavelength shift is $20 \mathrm{pm}$, the change of the recorded ratio can be detected clearly, which suggests that the resolution of the system is between 10 and $20 \mathrm{pm}$ (the theoretical prediction is $18 \mathrm{pm}$ ).

When the averaging value is increased to 128 , the fluctuation of the ratio is approximately $0.002 \mathrm{~dB}$ and the corresponding results are presented from Fig. $5(\mathrm{~g})$ to $5(\mathrm{i})$. In this case, the step change of the ratio can be detected clearly when the wavelength shift is
10 pm [see Fig. 5(g)], which means that the system's resolution is approximately $10 \mathrm{pm}$ when the fluctuation of the ratio caused by the noise of the photodetectors is approximately $0.002 \mathrm{~dB}$ (the theoretical result is $7 \mathrm{pm}$ ).

These experimental results agree with the above modeling results as shown in Fig. 3. Both the theoretical and experimental results show the influence of the noise of the photodetectors on the system's resolution. The detailed influence of the SNR of the input signal is not carried out experimentally because the SNR of the tunable laser is not controllable in our experiment. However, the prediction of system's resolution presented in Fig. 3 indicates that the input signal with a high SNR corresponds to a better wavelength resolution of the ratiometric system. It is because a high SNR allows a high slope of the transmission of the edge filter over a given wavelength range.

\section{Conclusion}

The resolution of a ratiometric wavelength measurement system has been investigated theoretically and experimentally. Theoretical modeling and analysis has shown that due to the signal-to-noise ratio of the input signal and the noise of the photodetectors associated with optical-to-electronic conversion, the resolution of a ratiometric wavelength measurement system is limited. A ratiometric system employing a macrobending standard single-mode fiber has been developed for the experimental verification. Corresponding results have been presented, which are in good agreement with the theoretical prediction.

The support of the Irish Research Council for Science, Engineering and Technology (IRCSET) is gratefully acknowledged.

\section{References}

1. B. Mason, S. P. DenBaars, and L. A. Coldren, "Tunable sampled-grating DBR lasers with integrated wavelength mornitors," IEEE Photon. Technol. Lett. 10, 1085-1087 (1998).

2. J. J. Lepley and A. S. Siddiqui, "Primary referenced DWDM frequency comb generator," IEE Proc. Optoelectron. 146, 121124 (1999)

3. A. D. Kersey, M. A. Davis, H. J. Patrick, M. LeBlanc, K. P. Koo, C. G. Askins, M. A. Putnam, and E. J. Friebele, "Fiber grating sensors," J. Lightwave. Technol. 15, 1442-1463 (1997).

4. S. M. Melle, K. Liu, and R. M. Measures, "A passive wavelength demodulation system for guided-wave Bragg grating sensors," IEEE Photon. Technol. Lett. 4, 516-518 (1992).

5. C. Sookdhis, T. Mei, H. S. Djie, and J. Arokiaraj, "Passive wavelength monitor based on multimode interference waveguide," Opt. Eng. 42, 3421-3422 (2003).

6. G. Z. Xiao, P. Zhao, F. G. Sun, Z. G. Lu, Z. Zhang, and C. P. Grover, "Interrogating fiber Bragg grating sensors by thermally scanning a demultiplexer based on arrayed waveguide gratings," Opt. Lett. 29, 2222-2224 (2004).

7. A. B. L. Ribeiro, L. A. Ferreira, M. Tsvekov, and J. L. Santos, "All-fiber interrogation technique for fiber Bragg sensors using a biconical fiber filter," Electron. Lett. 32, 382-383 (1996).

8. Y. Liu, L. Zhang, and I. Bennion, "Fabricating fibre edge filters with arbitrary spectral response based on tilted chirped grating structures,” Meas. Sci. Technol. 10, L1-L3 (1999). 
9. B. Mason, S. P. Denbarrs, and L. A. Coldren, "Tunable sampled-grating D. B. R. lasers with integrated wavelength monitors," IEEE Photon. Technol. Lett. 10, 1085-1087 (1998).

10. Q. Wang, G. Farrell, T. Freir, G. Rajan, and P. Wang, "Lowcost wavelength measurement based on a macrobending single-mode fiber," Opt. Lett. 31, 1785-1787 (2006).
11. Q. Wang and G. Farrell, "Multimode fiber based edge filter for optical wavelength measurement application," Microwave Opt. Technol. Lett. 48, 900-902 (2006).

12. Q. Wang, G. Farrell, and T. Freir, "Study of transmission response of edge filters employed in wavelength measurements," Appl. Opt. 44, 7789-7792 (2005). 